\title{
MicroRNA-15a-5p promotes the proliferation and invasion of T98G glioblastoma cells via targeting cell adhesion molecule 1
}

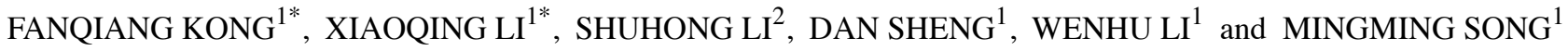 \\ Departments of ${ }^{1}$ Emergency and ${ }^{2}$ Obstetrics and Gynecology, \\ The Affiliated Yantai Yuhuangding Hospital of Qingdao University, Yantai, Shandong 264000, P.R. China
}

Received June 3, 2020; Accepted September 14, 2020

DOI: $10.3892 / \mathrm{ol} .2020 .12364$

\begin{abstract}
Glioblastoma (GBM) is a type of malignant tumor occurring in the brain that severely influences the life of affected individuals. GBM cells are highly infiltrative, which is one of the main obstacles in the treatment of the disease. Numerous microRNAs (miRNAs/miRs) are associated with the development of GBM. However, the effects of miR-15a-5p on GBM remain elusive. In the present study, reverse transcription-quantitative PCR and western blot analysis were applied for the detection of RNA and protein levels, respectively. Cell Counting Kit- 8 and Transwell assays were performed to examine cell proliferation and invasion, respectively. TargetScan 7.1 and dual-luciferase reporter assay were utilized for the prediction and verification of the association between miRNAs and mRNAs. The present study revealed that miR-15a-5p expression was upregulated in the GBM T98G cell line. The results further demonstrated that, through the inhibition of cell adhesion molecule 1 expression and the promotion of Akt phosphorylation, miR-15a-5p was able to promote GBM cell proliferation and invasion. Overall, the present findings revealed a novel mechanism responsible for the development of GBM and provided an experimental basis for the diagnosis and treatment of GBM.
\end{abstract}

\section{Introduction}

Glioblastoma (GBM) is the most common and severe type of human glioma, with an incidence rate of 3.19 cases/100,000 individuals/year globally, as reported in 2017 (1). Glioma is a primary malignant tumor occurring in the brain, the incidence rate of which is the highest among tumors of the central

Correspondence to: Dr Mingming Song, Department of Emergency, The Affiliated Yantai Yuhuangding Hospital of Qingdao University, East Road 20, Yantai, Shandong 264000, P.R. China E-mail: songmingmingqdu@yeah.net

${ }^{*}$ Contributed equally

Key words: glioblastoma, microRNA-15a-5p, cell proliferation, invasion, cell adhesion molecule 1 nervous system $(2,3)$. GBM is known as grade IV glioma, with characteristics of high malignancy, high recurrence rate and very low 5-year survival rate $(4-5 \%)(4,5)$. One of the reasons why GBM is difficult to cure is that the tumor tissue mainly grows infiltratively; the tumor cells migrate to the surrounding brain tissue during the process of proliferation, binding to the dura mater or directly penetrating into deep brain structures, such as the ventricles (6-8). The molecular mechanisms of GBM cell proliferation and invasion are crucial, and remain to be fully elucidated.

Some important molecules have been found to be involved in the proliferation and invasion of GBM cells, such as cell adhesion molecule 1 (CADM1) (9-11). CADM1 has been identified as a tumor suppressor in a variety of tumors, including lung, prostate, liver, pancreas and breast cancer (12). Studies have indicated that CADM1 is able to activate STAT3 and the $\mathrm{PI} 3 \mathrm{~K} / \mathrm{Akt}$ signaling pathway, and thus regulates the proliferation of tumor cells, including lung cancer cells and squamous cell carcinoma cells $(13,14)$. In GBM-associated research, it has been demonstrated that CADM1 expression is decreased in patients with GBM and in GBM cell lines, and CADM1 overexpression inhibits the proliferation of GBM cells $(9,11)$. These findings indicate that CADM1 is an effective suppressor of GBM proliferation.

MicroRNAs (miRNAs/miRs) are a type of non-coding RNAs, which form RNA-induced silencing complexes by binding to the mRNA of target genes, and thus inhibiting their translation $(15,16)$. Therefore, by regulating the expression levels of oncogenes and tumor suppressors, miRNAs are able to regulate the development and pathogenesis of a variety of tumors, including breast, prostate and pancreatic cancer (17). In addition, a number of studies have indicated that the expression levels of some miRNAs are abnormal during the occurrence of GBM (18-20). Therefore, miRNAs have been widely investigated in tumor detection and treatment, including GBM. For instance, the expression levels of miR-21 and $\mathrm{miR}-10 \mathrm{~b}$ are upregulated in GBM, while those of miR-15b, miR-137 and miR-124 are downregulated in GBM; additionally, manipulating the levels of these miRNAs can regulate some of the pathological characteristics of GBM, including cell migration, invasion, proliferation and apoptosis (21-24). Among the cancer-associated miRNAs, miR-15a-5p has been reported to be involved in the proliferation and invasion of a variety of tumor cells, including non-small cell lung 
cancer, colorectal adenocarcinoma and endometrial cancer cells (25-27). However, its role in GBM has not yet been elucidated. Therefore, the present study aimed to investigate the role of miR-15a-5p in GBM.

\section{Materials and methods}

Antibodies and reagents. The antibodies used in the present were as follows: CADM1 (1:1,000; cat. no. ABT66; EMD Millipore), Akt (1:1,000; cat. no. 9272; Cell Signaling Technology, Inc.), phosphorylated (p)-Akt (1:1,000; cat. no. 4060; Cell Signaling Technology, Inc.) and GAPDH (1:1,000; cat. no. HPA061280; EMD Millipore) primary antibodies, and goat anti-rabbit (1:3,000; cat. no. 7074; Cell Signaling Technology, Inc.) secondary antibodies.

The reagents used were as follows: Fetal bovine serum (FBS; Thermo Fisher Scientific, Inc.), Dulbecco's modified Eagle's medium (DMEM; Thermo Fisher Scientific, Inc.), penicillin-streptomycin (Thermo Fisher Scientific, Inc.), Lipofectamine $^{\circledR} 2000$ (Thermo Fisher Scientific, Inc.), TRIzol ${ }^{\circledR}$ (Thermo Fisher Scientific, Inc.), the PrimeScript qRT Reagent kit (Takara Bio, Inc.), SYBR-Green Mix (Roche Diagnostics), the Dual-Luciferase Assay System (Promega Corporation), Cell Counting Kit-8 (CCK-8; Dojindo Molecular Technologies, Inc.), protease inhibitor cocktail (Sigma-Aldrich; Merck KGaA), RIPA buffer (Roche Diagnostics), BSA (Beijing Solarbio Science \& Technology Co., Ltd.) and Premixed Luminata $^{\mathrm{TM}}$ Western HRP substrates for stronger signals (EMD Millipore).

Cell culture and transfection. The T98G cell line was obtained from the American Type Culture Collection. Human brain normal astroglia HEB cells were purchased from Ningbo Mingzhou Biotechnology Co., Ltd. (cat. no. MZ-0831). The cells were cultured in DMEM containing 10\% FBS and 1\% penicillin-streptomycin, and incubated in a $37^{\circ} \mathrm{C}$ incubator containing $5 \% \mathrm{CO}_{2}$.

Small RNAs $50 \mathrm{nM}$ miR-negative control (NC) mimic or miR-15a-5p mimic; $100 \mathrm{nM}$ miR-NC inhibitor or miR-15a-5p inhibitor] and small interfering (si)RNAs (50 $\mathrm{nM}$ siNC, siCADM1-1 and siCADM1-2) were transfected into the cells using Lipofectamine 2000. After $48 \mathrm{~h}$ of incubation, the cells were used for subsequent experiments. The non-targeting scrambled miR-NC mimic and miR-NC inhibitor, miR-15a-5p inhibitor and miR-15a-5p mimic were obtained from Shanghai GenePharma Co., Ltd., while siCADM1 and siNC were purchased from Shanghai Tuoran Biological Technology Co., Ltd. Their sequences were as follows: miR-NC inhibitor, 5'-CAGUACUUUUGUGUAGUACAA-3'; miR-15a-5p inhibitor, 5'-CACAAACCAUUAUGUGCUGCUA-3'; miR-NC mimic, 5'-UUCUCCGAACGUGUCACGUTT-3'; miR-15a-5p mimic sense, 5'-UAGCAGCACAUAAUGGUUUGUG-3' and antisense, 5'-CACAAACCAUUAUGUGCUGCUA-3'; siNC sense, 5'-UUCUCCGAACGUGUCACGUdTdT-3' and antisense, 5'-ACGUGACACGUUCGGAGA AdTdT-3'; siCADM1-1 sense, 5'-CCAACCUGUUCAUCAAUAAdTdT-3' and antisense, 5'-UUAUUGAUGAACAGGUUGGdTdT-3'; and siCADM1-2 sense, 5'-UCAGGUGGUUCAAAGGGA AdTdT-3' and antisense, 5'-UUCCCUUUGAACCACCUG AdTdT-3'.
Reverse transcription-quantitative PCR (RT-qPCR). Total RNA was extracted from the cultured T98G or HEB cells using TRIzol reagent, and then reverse transcribed into cDNA at $37^{\circ} \mathrm{C}$ for $15 \mathrm{~min}$ and $85^{\circ} \mathrm{C}$ for $5 \mathrm{sec}$ using the PrimeScript qRT Reagent kit according to the manufacturer's protocol. The SYBR-Green Mix kit was used for the RT-qPCR experiments on an ABI Prism 7500 system (Thermo Fisher Scientific, Inc.) according to the manufacturer's protocol. The thermocycling conditions were as follows: $95^{\circ} \mathrm{C}$ for $3 \mathrm{~min}$, followed by 40 cycles of $95^{\circ} \mathrm{C}$ for $10 \mathrm{sec}, 60^{\circ} \mathrm{C}$ for $15 \mathrm{sec}$ and $72^{\circ} \mathrm{C}$ for $31 \mathrm{sec}$. The expression levels of miRNAs and mRNAs were analyzed using the $2^{-\Delta \Delta C q}$ method (28), with U6 and GAPDH as the internal control for miRNAs and mRNAs, respectively. The primer sequences were as follows: miR-15a-5p forward, 5'-TAGCAGCACATAATGGTTTGTG-3' and reverse, 5'-CTC AACTGGTGTCGTGGA-3'; U6 forward, 5'-CTCGCTTCG GCAGCACA-3' and reverse, 5'-AACGCTTCACGAATTTGC GT-3'; CADM1 forward, 5'-CCACAGGTGATGGGCAGA AT-3' and reverse, 5'-TTCCTGTGGGGGATCGGTAT-3'; GAPDH forward, 5'-GAGAAGGCTGGGGCTCATTT-3' and reverse, 5'-AGTGATGGCATGGACTGTGG-3'.

Transwell assay. T98G cells $\left(5 \times 10^{4}\right)$ were seeded in serum-free DMEM in the upper chamber of a Matrigel-precoated Transwell chamber (Corning, Inc.), while the lower chamber was filled with DMEM with $10 \%$ FBS. Following incubation at $37^{\circ} \mathrm{C}$ for $24 \mathrm{~h}$, the upper chamber was removed, the polycarbonate membrane was inverted and cells on top of the membrane were removed using a cotton swab. The invading cells were fixed with $4 \%$ paraformaldehyde for $1 \mathrm{~h}$ at room temperature and stained with $0.25 \%$ crystal violet for $1 \mathrm{~h}$ at room temperature. The invading cells were counted based on five field digital images taken at a magnification of x100 using an Olympus IX51 light microscope (Olympus Corporation).

CCK- 8 assay. CCK- 8 assay was used to detect the cell proliferative ability. T98G cells transfected with miR-NC inhibitor, miR-15a-5p inhibitor, siNC or siCADM1 were seeded (1,000 cells/well) in 96-well plates and incubated at $37^{\circ} \mathrm{C}$ for different periods of time $(24,48$ or $72 \mathrm{~h})$. Prior to CCK- 8 detection, $100 \mu$ l fresh DMEM containing $10 \mu$ l CCK- 8 reagent were added to each well according to the manufacturer's protocol and the cells were cultured for $4 \mathrm{~h}$ at $37^{\circ} \mathrm{C}$. The OD values of the different groups of cells were measured at $450 \mathrm{~nm}$ using a spectrophotometer (BioTek Instruments, Inc.).

Dual-luciferase reporter assay. The candidate target genes for miR-15a-5p were screened using TargetScan (http://www. targetscan.org) (29). The 3'-untranslated region (3'-UTR) sequences of wild-type (WT) CADM1 were obtained from T98G cell cDNA, and inserted into pGL3-luciferase reporter plasmids (Promega Corporation) using KpnI and XhoI restriction sites to obtain pGL3-CADM1-3'-UTR-WT. Two-point mutations were introduced into CADM1 3'-UTR using a site-directed mutagenesis kit (Agilent Technologies, Inc.), in order to construct pGL3-CADM1-3'-UTR-mutant (Mut). The plasmids were then co-transfected with $50 \mathrm{nM}$ miR-15a-5p mimic or NC mimic into $\mathrm{T} 98 \mathrm{G}$ cells using Lipofectamine 2000. After incubation at $37^{\circ} \mathrm{C}$ for $48 \mathrm{~h}$, the luciferase intensity was detected using the Dual-Luciferase Assay System 

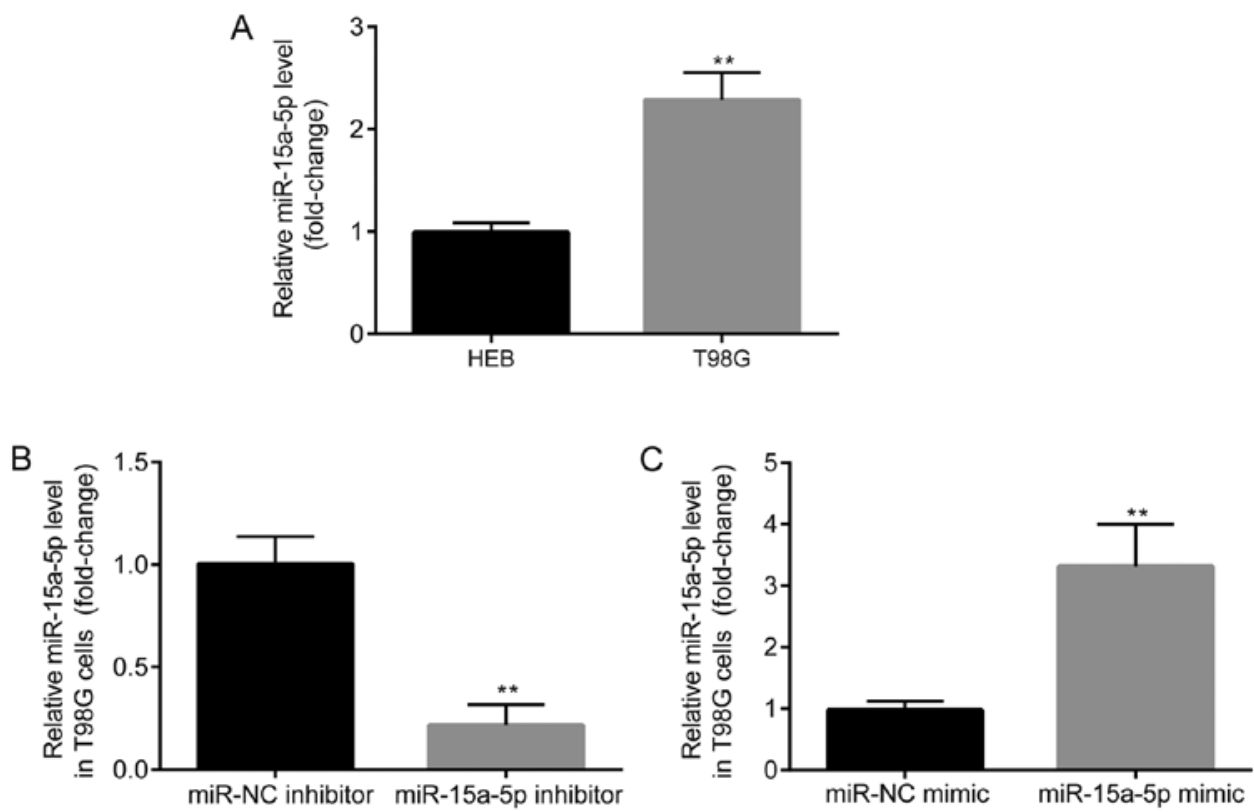

Figure 1. miR-15a-5p is upregulated in a glioblastoma cell line. (A) Reverse transcription-quantitative PCR revealed that miR-15a-5p expression was higher in T98G cells than in HEB cells. (B) miR-15a-5p inhibitor suppressed miR-15a-5p expression. (C) miR-15a-5p mimic increased miR-15a-5p expression. ${ }^{* *} \mathrm{P}<0.01$. miR, microRNA; NC, negative control.

(Promega Corporation). Renilla luciferase activity was used as the reference.

Western blot analysis. T98G cells were washed with PBS, collected in test tubes by centrifugation at $1,000 \mathrm{x}$ g at $4^{\circ} \mathrm{C}$ for $5 \mathrm{~min}$, and lysed with RIPA buffer containing protease inhibitor. Protein concentration was determined using a BCA kit (Beyotime Institute of Biotechnology). The SDS loading buffer was mixed with the protein samples. Proteins (15 $\mu \mathrm{g} /$ lane) were separated via $8 \%$ SDS-PAGE and transferred to a PVDF membrane, which was blocked with 5\% BSA at room temperature for $2 \mathrm{~h}$ in TBS-Tween 20 (0.5\%; TBST), then incubated with primary antibodies overnight at $4^{\circ} \mathrm{C}$, followed by HRP-conjugated secondary antibodies at room temperature for $1 \mathrm{~h}$. After washing thoroughly with TBST, the HRP signals were detected with chemical HRP substrate. The intensity of positive protein bands was measured using ImageJ software version 1.50 (National Institutes of Health), and the average value of the control group was normalized to 1. GAPDH served as the reference protein.

Statistical analysis. All data were analyzed using GraphPad prism 6.0 (GraphPad Software, Inc.) and are expressed as the mean \pm SEM. Each experiment was repeated $\geq 3$ times. Unpaired student's t-test was used to compare differences between 2 groups. One-way ANOVA followed by Tukey's post hoc test was used to compare differences among $\geq 3$ groups. $\mathrm{P}<0.05$ was considered to indicate a statistically significant difference.

\section{Results}

miR-15a-5p expression is upregulated in T98G cells. To examine whether miR-15a-5p expression is normal in GBM, RT-qPCR was performed to detect miR-15a-5p expression in
T98G and HEB cells. T98G is a widely used human GBM cell line, while HEB is a type of human brain normal astroglia cell line. As shown by the results presented in Fig. 1A, miR-15a-5p expression was significantly higher in T98G cells compared with that in HEB cells, which indicated that miR-15a-5p expression was upregulated in GBM cells. To further examine the roles of miR-15a-5p in GBM, miR-15a-5p inhibitor and miR-15a-5p mimic were designed to inhibit or enhance miR-15a-5p expression, respectively (Fig. 1B and C).

miR-15a-5p promotes $T 98 G$ cell proliferation and invasion. In order to verify whether miR-15a-5p participates in GBM cell proliferation and invasion, CCK-8 and Transwell assays were performed. As shown in Fig. 2A, the results of CCK-8 assay revealed that the proliferation of $\mathrm{T} 98 \mathrm{G}$ cells was significantly suppressed by the miR-15a-5p inhibitor compared with the NC inhibitor. Similarly, as shown by Transwell assay, which was used to measure the cell invasive ability, miR-15a-5p inhibitor effectively restrained the invasion of T98G cells (Fig. 2B and C). These experiments indicated that miR-15a-5p was able to promote the proliferation and invasion of GBM cells.

$C A D M 1$ is regulated by $m i R-15 a-5 p$. In order to determine the mechanisms through which miR-15a-5p regulates cell proliferation and invasion, the candidate target genes of miR-15a-5p were screened using TargetScan. The results revealed that the 3'-UTR of CADM1 may contain a targeting site of miR-15a-5p (Fig. 3A). For further verification, a normal CADM1 3'-UTR sequence (WT) and a mutated one (Mut) were designed, and each sequence was inserted into the luciferase reporter plasmid for dual-luciferase activity assay. As shown by the results presented in Fig. 3B, in the T98G cells transfected with the WT reporter plasmid, the luciferase activity in the miR-15a-5p mimic group was significantly lower than that in the NC mimic group. On the other hand, no significant 

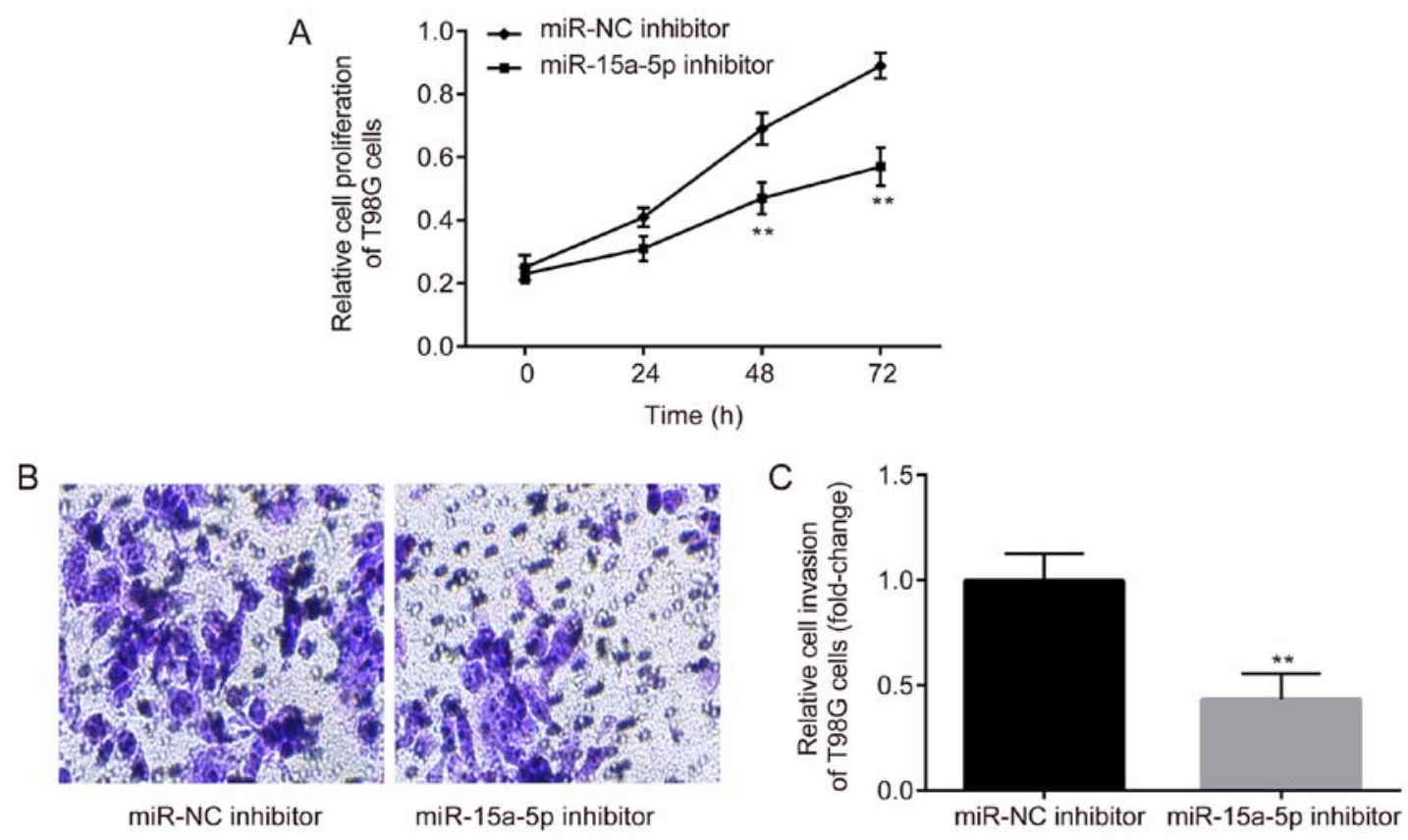

Figure 2. miR-15a-5p inhibitor suppresses T98G cell proliferation and invasion. (A) Cell Counting Kit- 8 assay revealed that the miR-15a-5p inhibitor suppressed T98G cell proliferation. (B) Transwell assay revealed that miR-15a-5p inhibitor suppressed T98G cell invasion (magnification, x100). (C) Quantification of Transwell assay showing a significant decrease in cell invasion in the miR-15a-5p inhibitor group. ${ }^{* *} \mathrm{P}<0.01 \mathrm{vs}$. miR-NC inhibitor. miR, microRNA; NC, negative control.

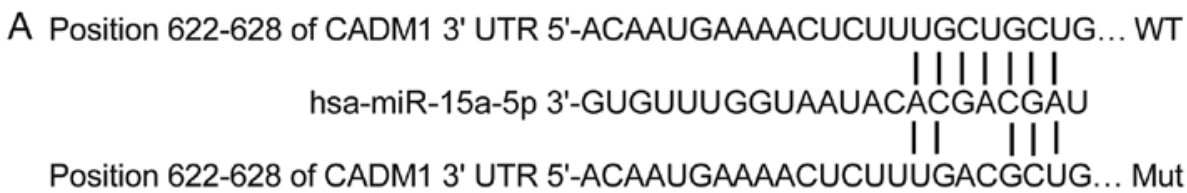

B

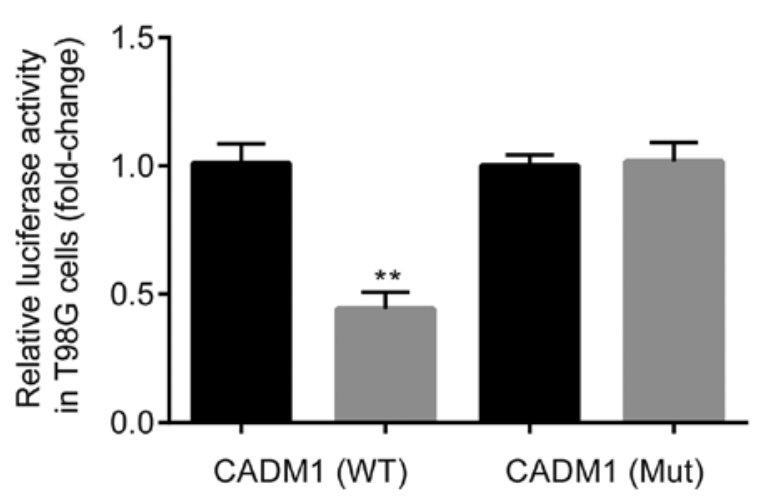

miR-NC mimic

miR-15a-5p mimic

Figure 3. miR-15a-5p targets CADM1. (A) WT and Mut sequences of the CADM1 3'-UTR. (B) Dual-luciferase assay revealed that the miR-15a-5p mimic suppressed luciferase activity in the CADM1 (WT) group, but not in the CADM1 (Mut) group. ${ }^{* *} \mathrm{P}<0.01 \mathrm{vs}$. miR-NC mimic. miR, microRNA; NC, negative control; WT, wild-type; Mut, mutant; CADM1, cell adhesion molecule 1; UTR, untranslated region.

difference was observed between the two groups transfected with the Mut plasmid.

Subsequently, the present study determined whether CADM1 expression was altered in GBM cells. RT-qPCR and western blot analysis were performed to detect the expression levels of CADM1 in T98G and HEB cells. The results revealed that both the mRNA and protein levels of CADM1 were downregulated in T98G cells compared with those in HEB cells (Fig. 4A-C). These findings were consistent with the upregulated miR-15a-5p phenotype observed in T98G cells.
Inhibiting CADM1 attenuates the effects of miR-15a-5p on cell proliferation and invasion. To examine whether CADM1 was involved in miR-15a-5p-induced cell proliferation and invasion, two CADM1 siRNAs (Fig. 5A and B) were designed, with siCADM-1 resulting more effective and being therefore selected for further research. Subsequently, siCADM1-1 and miR-15a-5p inhibitor were co-transfected into the T98G cells for CCK-8 and Transwell assays. As shown in Fig. 5C-E, miR-15a-5p inhibitor significantly suppressed $\mathrm{T} 98 \mathrm{G}$ cell proliferation and invasion, and 

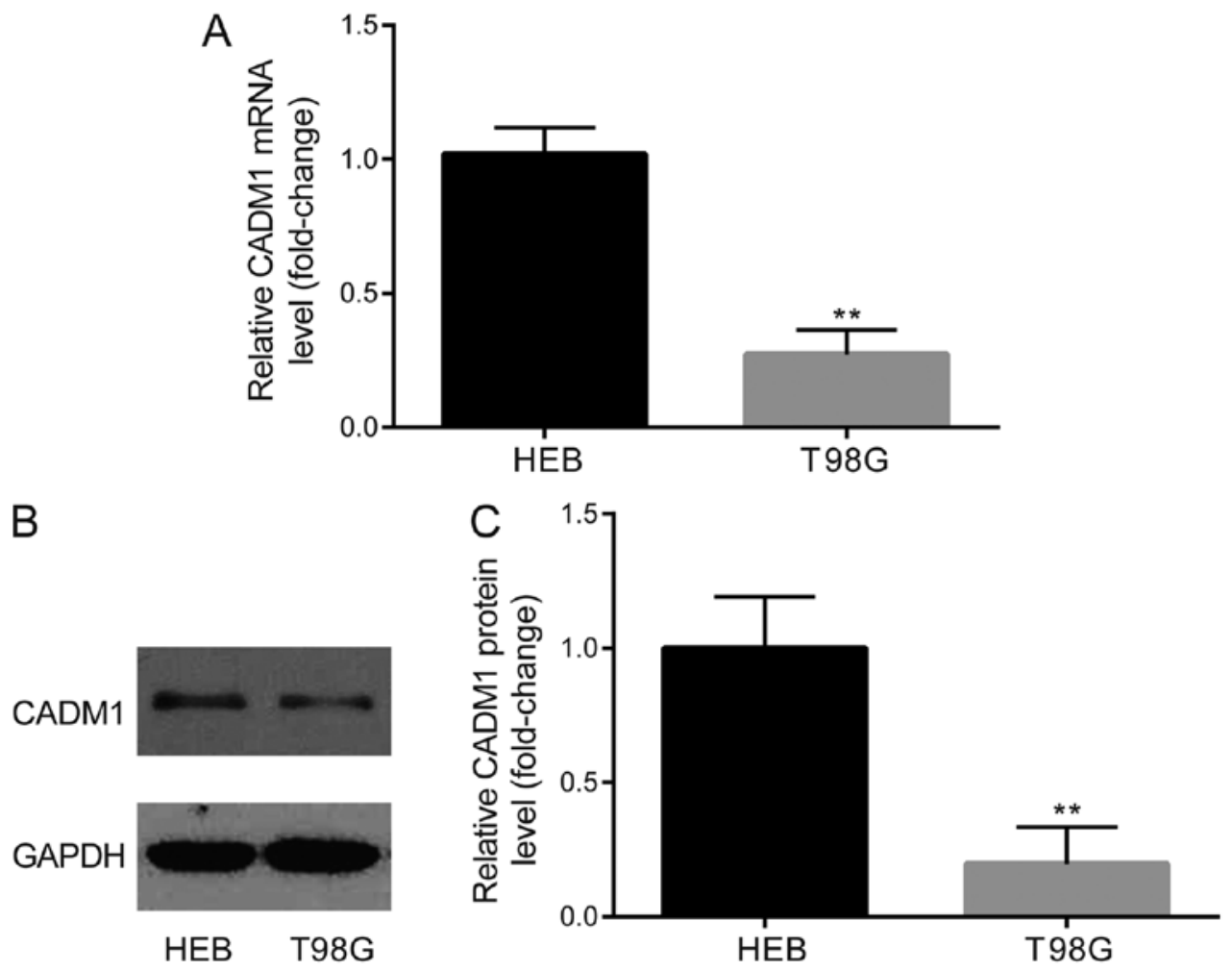

Figure 4. CADM1 expression is downregulated in T98G cells. (A) Reverse transcription-quantitative PCR revealed that the mRNA levels of CADM1 were lower in T98G cells than in HEB cells. (B) Western blot analysis revealed that the protein levels of CADM1 were lower in T98G cells than in HEB cells. (C) Quantification of western blot analysis showing a significant decrease in CADM1 protein expression in T98G cells. ** P<0.01 vs. HEB cells. CADM1, cell adhesion molecule 1 .

A

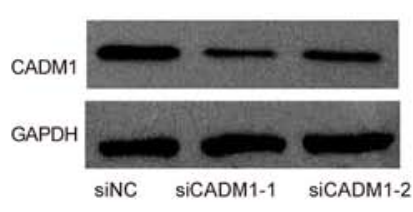

B

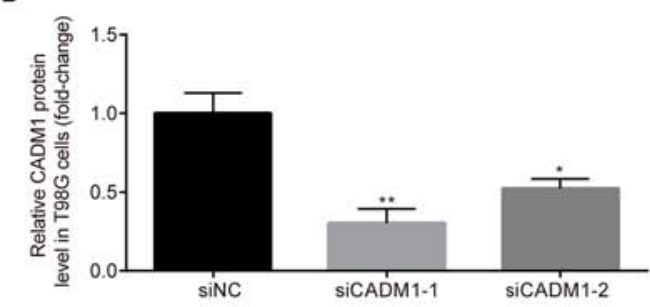

C $\rightarrow$ miR-NC inhibitor+sinc

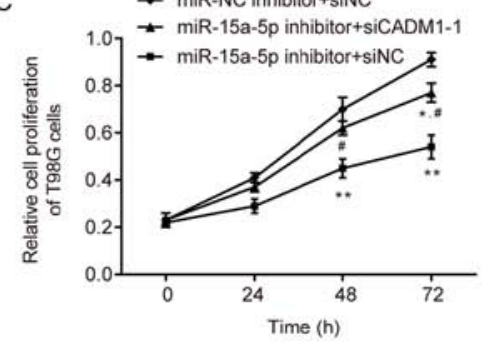

D

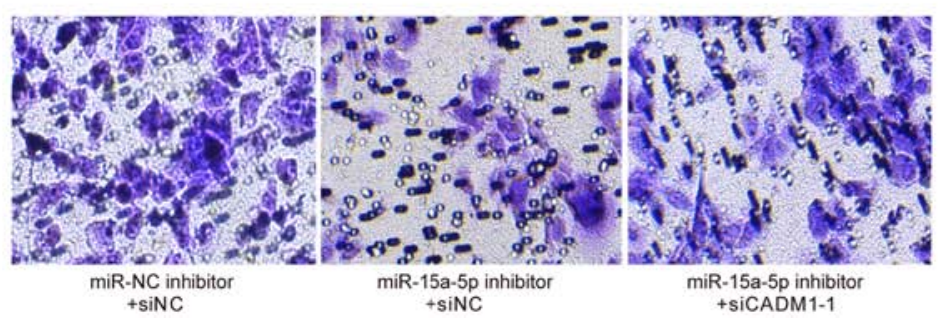

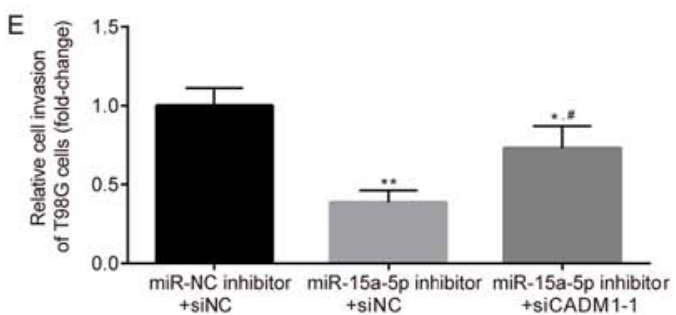

Figure 5. CADM1 knockdown reverses the inhibitory effects of miR-15a-5p inhibitor on T98G cell proliferation and invasion. (A) Western blot analysis revealed that the protein levels of CADM1 were downregulated by CADM1 siRNAs. (B) Quantification of western blot analysis showing a significant decrease in CADM1 protein expression. ${ }^{*} \mathrm{P}<0.05$ and ${ }^{* *} \mathrm{P}<0.01$ vs. siNC. (C) Cell Counting Kit- 8 assay revealed that siCADM1-1 attenuated the inhibitory effect of miR-15a-5p inhibitor on T98G cell proliferation. (D) Transwell assay revealed that siCADM1-1 attenuated the inhibitory effect of miR-15a-5p inhibitor on T98G cell invasion (magnification, x100). (E) Quantification of Transwell assay showing a significant decrease in cell invasion in the miR-15a-5p inhibitor group compared with in the control group, and a significant increase in the miR-15a-5p inhibitor plus siCADM1-1 group compared with in the miR-15a-5p inhibitor group. ${ }^{*} \mathrm{P}<0.05$ and ${ }^{* *} \mathrm{P}<0.01$ vs. miR-NC inhibitor + siNC; ${ }^{\#} \mathrm{P}<0.05$ vs. miR-15a-5p inhibitor + siNC. miR, microRNA; NC, negative control; CADM1, cell adhesion molecule 1; si, small interfering.

these phenotypes were attenuated when the cells were co-transfected with siCADM1-1. These results suggested that miR-15a-5p promoted T98G cell proliferation and invasion by targeting CADM1.
Effects of miR-15a-5p and CADM1 on Akt activity. Akt, also known as protein kinase $B$, is an important protein regulating cell proliferation and has been found to be overactivated in GBM tissues and cells $(30,31)$. p-Akt is the 
A

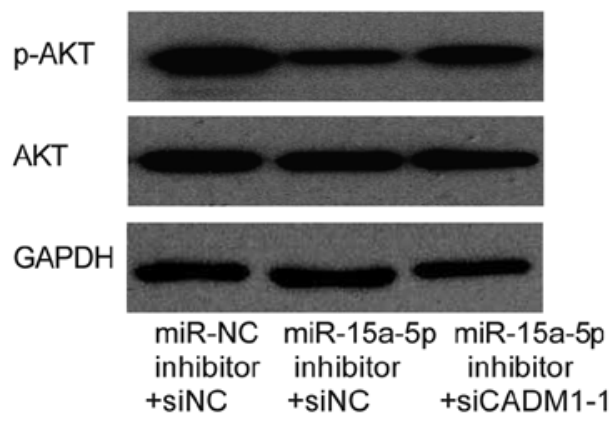

B

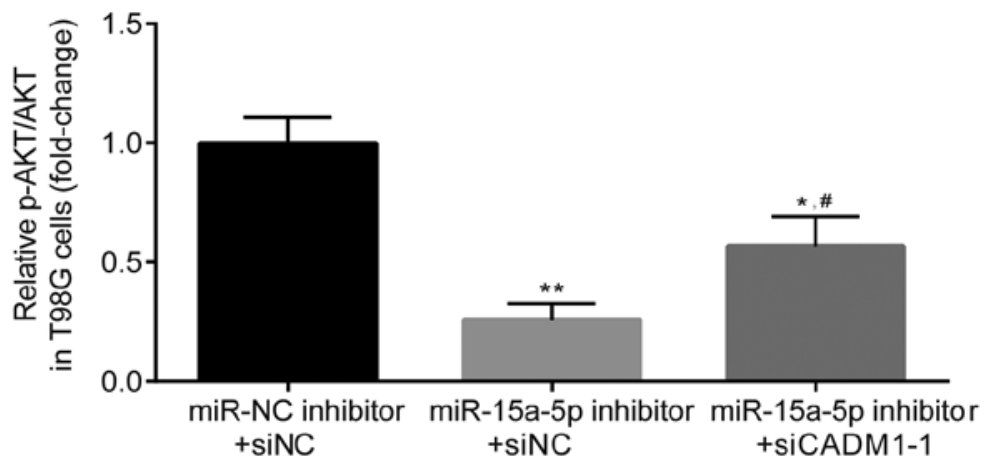

Figure 6. CADM1 knockdown reverses the inhibitory effects of the miR-15a-5p inhibitor on Akt phosphorylation. (A) Western blot analysis revealed that Akt phosphorylation was suppressed using the miR-15a-5p inhibitor, which was reversed using siCADM1-1. (B) Quantification of western blot analysis showing a significant decrease in Akt phosphorylation levels in the miR-15a-5p inhibitor group compared with in the control groups, and a significant increase in the miR-15a-5p inhibitor plus siCADM1-1 group compared with in the miR-15a-5p inhibitor group. ${ }^{*} \mathrm{P}<0.05$ and ${ }^{* * *} \mathrm{P}<0.01$ vs. miR-NC inhibitor + siNC; ${ }^{\#} \mathrm{P}<0.05$ vs. miR-15a-5p inhibitor + siNC. miR, microRNA; NC, negative control; CADM1, cell adhesion molecule 1; si, small interfering; p, phosphorylated.

activated form, and has been demonstrated to be regulated by CADM1 (13). Consequently, the present study investigated whether miR-15a-5p and CADM1 were able to regulate Akt phosphorylation in T98G cells. As shown in Fig. 6A and B, the phosphorylation levels of Akt were decreased using the miR-15a-5p inhibitor, and this effect was partially reversed by siCADM1-1.

\section{Discussion}

The present study identified a novel mechanism through which miRNAs may promote GBM cell proliferation and invasion. The data demonstrated that miR-15a-5p expression was higher in the GBM T98G cell line than in an immortal control cell line. Introducing miR-15a-5p inhibitor into T98G cells effectively inhibited Akt phosphorylation and suppressed T98G cell proliferation and invasion. The cell adhesion protein CADM1 was found to be regulated by miR-15a-5p, and inhibiting CADM1 in the T98G cells attenuated the effects induced by the miR-15a-5p inhibitor.

One of the key findings of the present study was that miR-15a-5p expression in T98G cells was significantly increased compared with that in normal cells, and subsequent experiments revealed that miR-15a-5p inhibition suppressed cell proliferation and invasion, indicating that miR-15a-5p was able to promote cancer cell invasion. Therefore, in GBM cells, the abnormal expression levels of miR-15a-5p further aggravated the cancer pathological process. In addition, miR-15a-5p expression has been evaluated in other types of tumors, including non-small cell lung cancer and colorectal adenocarcinoma, in which it has been found to promote cancer cell proliferation $(25,26)$. However, a previous study has reported that miR-15a-5p expression is decreased in endometrial carcinoma (27), suggesting that the role of miR-15a-5p may differ in various types of cancer.

The present study identified that CADM1 was one of the candidate target genes of miR-15a-5p using TargetScan, and this finding was confirmed using the dual-luciferase activity assay. The current results demonstrated that only the miR-15a-5p mimic, but not the NC mimic or Mut CADM1 3'-UTR, suppressed luciferase activity. This strongly suggested that CADM1 may be targeted by miR-15a-5p. In addition, subsequent experiments revealed a functional association between miR-15a-5p and CADM1. In previous studies, CADM1 has been demonstrated to interact with FERM and PDZ domain containing protein and inhibit cancer cell proliferation of squamous cell carcinoma and non-small cell lung cancer $(14,32)$. In GBM-associated studies, it has been demonstrated that CADM1 expression in GBM cell lines and patients with GBM are significantly decreased (9). These findings are consistent with the results of the present study, suggesting that CADM1 may be a reliable and stable tumor suppressor.

In addition to CADM1, there are several other target genes of miR-15a-5p. It has been reported that miR-15a-5p regulates the proliferation of endometrial mesenchymal stem cells by inhibiting VEGFA (33), and can also inhibit the growth of uterine leiomyoma cells by inhibiting the Wnt 3 protein through the Wnt/ $\beta$-catenin signaling pathway (27). These results suggest that miR-15a-5p may be able to regulate cell cycle through different signaling pathways. CADM1 inhibition alone may not be sufficient to reverse all the effects induced by the miR-15a-5p inhibitor, which was one of the reasons that the knockdown of CADM1 expression only partially reversed the effects of the miR-15a-5p inhibitor on T98G cell proliferation and invasion. Another reason for this partial reversal effect may be that a small amount of CADM1 is sufficient to inhibit cancer cell proliferation and invasion, since siCADM1 could not completely prevent all CADM1 mRNA translation.

The PI3K/Akt signaling pathway is closely associated with the proliferation and invasion of a number of tumor cells, including GBM and non-small cell lung cancer cells $(30,31,34)$. The present study firstly demonstrated that miR-15a-5p was able to inhibit Akt activity in GBM cells, which was consistent with the results obtained in other cancer systems, including gastric and ovarian cancer $(35,36)$. Additionally, it has been demonstrated that Akt participates in other physiological processes of tumor cells, such as autophagy, aging and apoptosis (37-39), suggesting that miR-15a-5p may also serve a role in these functions.

In conclusion, the present study demonstrated that miR-15a-5p promoted GBM cell proliferation and invasion by targeting CADM1. The present findings revealed a novel 
candidate mechanism through which miRNAs may participate in the development of GBM and provided a potential target for the treatment of GBM.

\section{Acknowledgements}

Not applicable.

\section{Funding}

No funding was received.

\section{Availability of data and materials}

The datasets generated and/or analyzed during the current study are not publicly available due to their association with another ongoing study, but are available from the corresponding author on reasonable request.

\section{Authors' contributions}

FK, XL, SL, DS and WL conducted the experiments and data analysis. MS designed the study and wrote the article. All authors read and approved the final manuscript.

\section{Ethics approval and consent to participate}

Not applicable.

\section{Patient consent for publication}

Not applicable.

\section{Competing interests}

The authors declare that they have no competing interests.

\section{References}

1. Batash R, Asna N, Schaffer P, Francis N and Schaffer M: Glioblastoma multiforme, diagnosis and treatment; recent literature review. Curr Med Chem 24: 3002-3009, 2017.

2. Ricard D, Idbaih A, Ducray F, Lahutte M, Hoang-Xuan K and Delattre JY: Primary brain tumours in adults. Lancet 379 : 1984-1996, 2012.

3. Dalessio A, Proietti G, Sica G and Scicchitano BM: Pathological and molecular features of glioblastoma and its peritumoral tissue. Cancers (Basel) 11: 469, 2019.

4. Louis DN, Perry A, Reifenberger G, von Deimling A Figarella-Branger D, Cavenee WK, Ohgaki H, Wiestler OD, Kleihues P and Ellison DW: The 2016 World health organization classification of tumors of the central nervous system: A summary. Acta Neuropathol 131: 803-820, 2016.

5. Carlsson SK, Brothers SP and Wahlestedt D: Emerging treatment strategies for glioblastoma multiforme. EMBO Mol Med 6: $1359-1370,2014$

6. Aldape KD, Brindle KM, Chesler L, Chopra R, Gajjar A, Gilbert MR, Gottardo N, Gutmann DH, Hargrave D, Holland EC, et al: Challenges to curing primary brain tumours Nat Rev Clin Oncol 16: 509-520, 2019.

7. Brandao M, Simon T, Critchley G and Giamas G: Astrocytes, the rising stars of the glioblastoma microenvironment. Glia 67: 779-790, 2019.

8. Osti D, De Bene MD, Rappa G, Santos M, Matafora V, Richichi C, Faletti S, Beznoussenko GV, Mironov A, Bachi A, et al: Clinical significance of extracellular vesicles in plasma from glioblastoma patients. Clin Cancer Res 25: 266-276, 2019.
9. Isobe T, Hisamori S, Hogan DJ, Zabala M, Hendrickson DG, Dalerba P, Cai S, Scheeren F, Kuo AH, Sikandar SS, et al: miR-142 regulates the tumorigenicity of human breast cancer stem cells through the canonical WNT signaling pathway. Elife 3: e01977, 2014.

10. Ghosh D, Ulasov IV, Chen L, Harkins LE, Wallenborg K, Hothi P, Rostad S, Hood L and Cobbs CS: TGF $\beta$-responsive HMOX1 expression is associated with stemness and invasion in glioblastoma multiforme. Stem Cells 34: 2276-2289, 2016.

11. Zhang X, Li W, Kang Y, Zhang J and Yuan H: SynCAM, a novel putative tumor suppressor, suppresses growth and invasiveness of glioblastoma. Mol Biol Rep 40: 5469-5475, 2013.

12. Murakami Y: Involvement of a cell adhesion molecule, TSLC1/IGSF4, in human oncogenesis. Cancer Sci 96: 543-552, 2005.

13. Kikuchi S, Iwai M, Sakurai-Yageta M, Tsuboi Y, Ito T, Maruyama T, Tsuda H, Kanai Y, Onizuka M, Sato Y and Murakami Y: Expression of a splicing variant of the CADM1 specific to small cell lung cancer. Cancer Sci 103: 1051-1057, 2012.

14. Vallath S, Sage EK, Kolluri KK, Lourenco SN, Teixeira VS, Chimalapati S, George PJ, Janes SM and Giangreco A: CADM1 inhibits squamous cell carcinoma progression by reducing STAT3 activity. Sci Rep 6: 24006, 2016.

15. Gregory RI, Chendrimada TP and Shiekhattar R: MicroRNA biogenesis: Isolation and characterization of the microprocessor complex. Methods Mol Biol 342: 33-47, 2006.

16. Krol J, Loedige I and Filipowicz W: The widespread regulation of microRNA biogenesis, function and decay. Nat Rev Genet 11: 597-610, 2010.

17. Lee YS and Dutta A: MicroRNAs in cancer. Annu Rev Pathol 4: 199-227, 2009.

18. Dong Z and Cui H: Epigenetic modulation of metabolism in glioblastoma. Semin Cancer Biol 57: 45-51, 2019.

19. Candido S, Lupo G, Pennisi M, Basile MS, Anfuso CD Petralia MC, Gattuso G, Vivarelli S, Spandidos DA, Libra M and Falzone L: The analysis of miRNA expression profiling datasets reveals inverse microRNA patterns in glioblastoma and Alzheimer's disease. Oncol Rep 42: 911-922, 2019.

20. Ye X, Wei W, Zhang Z, He C, Yang R, Zhang J, Wu Z, Huang Q and Jiang Q: Identification of microRNAs associated with glioma diagnosis and prognosis. Oncotarget 8: 26394-26403, 2017.

21. Guessous F, Alvaradovelez M, Marcinkiewicz L, Zhang Y, Kim J, Heister S, Kefas B, Godlewski J, Schiff D, Purow B and Abounader R: Oncogenic effects of miR-10b in glioblastoma stem cells. J Neurooncol 112: 153-163, 2013.

22. Wang J, Liu H, Tian L, Wang F, Han L, Zhang W and Bai YA: miR-15b inhibits the progression of glioblastoma cells through targeting insulin-like growth factor receptor 1 . Horm Cancer 8: 49-57, 2017

23. Shi L, Chen J, Yang J, Pan T, Zhang S and Wang Z: MiR-21 protected human glioblastoma U87MG cells from chemotherapeutic drug temozolomide induced apoptosis by decreasing Bax/Bcl-2 ratio and caspase-3 activity. Brain Res 1352: 255-264, 2010.

24. Silber J, Lim DA, Petritsch C, Persson AI, Maunakea AK, Yu M, Vandenberg SR, Ginzinger DG, James CD, Costello JF, et al: miR-124 and miR-137 inhibit proliferation of glioblastoma multiforme cells and induce differentiation of brain tumor stem cells. BMC Med 6: 14, 2008.

25. Ergun S, Güney S, Temiz E, Petrovic N and Gunes S: Significance of miR-15a-5p and CNKSR3 as novel prognostic biomarkers in non-small cell lung cancer. Anticancer Agents Med Chem 18: $1695-1701,2018$

26. Kontos CK, Tsiakanikas P, Avgeris M, Papadopoulos IN and Scorilas A: miR-15a-5p, a novel prognostic biomarker, predicting recurrent colorectal adenocarcinoma. Mol Diagn Ther 21: 453-464, 2017.

27. Wang ZM, Wan XH, Sang GY, Zhao JD and Wang DM: miR-15a-5p suppresses endometrial cancer cell growth via Wnt/ $\beta$-catenin signaling pathway by inhibiting WNT3A. Eur Rev Med Pharmacol Sci 21: 4810-4818, 2017.

28. Livak KJ and Schmittgen TD: Analysis of relative gene expression data using real-time quantitative PCR and the 2(-Delta Delta C(T)) method. Methods 25: 402-408, 2001.

29. Lewis BP, Burge CB and Bartel DP: Conserved seed pairing, often flanked by adenosines, indicates that thousands of human genes are microRNA targets. Cell 120: 15-20, 2005.

30. Karar J and Maity A: PI3K/AKT/mTOR pathway in angiogenesis. Front Mol Neurosci 4: 51, 2011 
31. Li X, Wu C, Chen N, Gu H, Yen A, Cao L, Wang E and Wang L: PI3K/Akt/mTOR signaling pathway and targeted therapy for glioblastoma. Oncotarget 7: 33440-33450, 2016.

32. Mao X, Seidlitz E, Ghosh K, Murakami Y and Ghosh HP: The cytoplasmic domain is critical to the tumor suppressor activity of TSLC1 in non-small cell lung cancer. Cancer Res 63: 7979-7985, 2003.

33. Chen $\mathrm{H}$ and Tian Y: MiR-15a-5p regulates viability and matrix degradation of human osteoarthritis chondrocytes via targeting VEGFA. Biosci Trends 10: 482-488, 2017.

34. Vivanco I and Sawyers CL: The phosphatidylinositol 3-Kinase AKT pathway in human cancer. Nat Rev Cancer 2: 489-501, 2002.

35. Pang K, Song J, Bai Z and Zhang Z: miR-15a-5p targets PHLPP2 in gastric cancer cells to modulate platinum resistance and is a suitable serum biomarker for oxaliplatin resistance. Neoplasma 67: 1114-1121, 2020.

36. Zhang K, Zhong W, Li WP, Chen ZJ and Zhang C: miR-15a-5p levels correlate with poor ovarian response in human follicular fluid. Reproduction 154: 483-496, 2017.
37. Franke TF, Hornik CP, Segev L, Shostak GA and Sugimoto C: PI3K/Akt and apoptosis: Size matters. Oncogene 22: 8983-8998, 2003.

38. Mirzaa GM, Campbell CD, Solovieff N, Goold C, Jansen LA, Menon S, Timms AE, Conti V, Biag JD, Adams C, et al: Association of MTOR mutations with developmental brain disorders, including megalencephaly, focal cortical dysplasia, and pigmentary mosaicism. JAMA Neurol 73: 836-845, 2016.

39. Yang J, Pi C and Wang G: Inhibition of PI3K/Akt/mTOR pathway by apigenin induces apoptosis and autophagy in hepatocellular carcinoma cells. Biomed Pharmacother 103: 699-707, 2018.

(i) (9) This work is licensed under a Creative Commons EY No ND Attribution-NonCommercial-NoDerivatives 4.0 International (CC BY-NC-ND 4.0) License. 